\title{
Dengue en un neonato
}

\author{
Kovy Arteaga-Livias, Angela Bonilla-Crispin, Vicky Panduro-Correa, \\ Carlos Martínez-Enríquez y Bernardo Dámaso-Mata
}

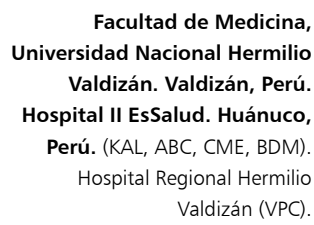

Conflictos de interés: Los autores no declaran ningún conflicto de

Fuente de financiamiento: Autofinanciado

Recibido: 7 de marzo de 2017 Aceptado: 8 de agosto de 2017

Correspondencia a: Kovy Arteaga-Livias hcoper@hotmail.com

\section{Introducción}

$\mathrm{E}$ 1 dengue es la enfermedad viral transmitida por artrópodos de mayor importancia en el mundo; y si bien no se limita a ellos, afecta especialmente a países tropicales. La enfermedad es producida por el virus del dengue (DENV), constituida por cuatro serotipos: DENV-1, DENV-2, DENV-3 y DENV-4. Pertenece al género flavivirus, y es transmitido por mosquitos, principalmente por Aedes aegypti ${ }^{1}$.

Se estima que alrededor del mundo, anualmente existen 390 millones de infectados, principalmente en países en vías de desarrollo. Aunque la mayoría de los casos son asintomáticos o solamente causan un cuadro febril inespecífico, algunos pacientes pueden desarrollar cuadros graves, shock, falla orgánica e incluso la muerte ${ }^{2}$.

En Latinoamérica, el dengue continúa siendo un problema de salud pública. Entre los años 2003 y 2013 se quintuplicaron los casos notificados. Entre 2009 y 2012 fueron notificados más de un millón de casos anuales, llegando a alcanzar en el año 2013 más de 2,3 millones de casos $^{3}$. Situación similar vive el Perú, donde se observa que desde el año 2008 los casos anuales notificados entre probables y confirmados están sobre 10.000 , con un máximo en el año 2015, cuando se llegó a un total de 35.816 casos confirmados ${ }^{4}$.

En algunas regiones del mundo, el dengue se ha convertido en una importante causa de morbilidad para la población infantil. En Asia, la enfermedad se encuentra entre las 10 principales causas de hospitalización y muerte ${ }^{5}$. La prevalencia de la enfermedad en la niñez es diferente según el grupo etario; así, por ejemplo, en el sudeste asiático la incidencia de la enfermedad fue de 0,5 por cada mil niños entre los tres y ocho meses de vida ${ }^{6}$.

Durante la gestación, el dengue se ha asociado a parto prematuro y bajo peso al nacer, observados principalmente cuando la enfermedad ha sido sintomática o grave en la gestante ${ }^{7}$.

El dengue en la etapa neonatal está pobremente estudiado, con escasos reportes de casos y series pequeñas, tanto de transmisión vertical probada o probable o bien vectorial. Se desconoce la fisiopatología de la enfermedad en este grupo etario, así como si la enfermedad tiene mayor gravedad que en la población adulta ${ }^{8}$.

Presentamos el caso de un recién nacido con diagnóstico de dengue.

\section{Caso clínico}

Paciente varón, nacido a las 40 semanas de gestación, mediante una cesárea programada, por madre con cesáreas previas y macrosomía fetal, con peso al nacer de $4.200 \mathrm{~g}$. A los 12 días de vida presentó fiebre e irritabilidad, por lo que fue llevado a un hospital en la selva central del Perú, donde llegó con fiebre cuantificada en $38,7^{\circ} \mathrm{C}$, frecuencia cardíaca: 144 por min, 55 respiraciones por min y $95 \%$ de saturación según oximetría de pulso. Se realizó una evaluación física completa, donde no se encontró ningún hallazgo significativo, exceptuando que presentaba llanto e irritabilidad, fontanela a tensión normal, sin otra afectación en el examen neurológico.

La madre de 39 años, multípara, embarazo controlado, sin episodios previos de dengue, tenía el antecedente 
de un cuadro febril al cuarto día después del parto, con calofríos, dolor retro-ocular, mialgias, hipersomnia y un rash generalizado. Por plaquetopenia progresiva se internó con el diagnóstico de dengue con signos de alarma, con prueba rápida e IgM positiva, dándose de alta cuatro días después.

Al RN se le realizó un hemograma que se encontraba dentro de límites normales, con una proteína $\mathrm{C}$ reactiva (PCR) cualitativa positiva. Se internó con el diagnóstico de sepsis neonatal, iniciándose terapia con cefotaxima $150 \mathrm{mg} / \mathrm{kg} /$ día y ampicilina $200 \mathrm{mg} / \mathrm{kg} /$ día, indicándose además continuar con lactancia materna.

Al día siguiente del ingreso, el paciente persistió febril, con plaquetopenia de 60.000 céls $/ \mathrm{mm}^{3}$, pero sin mayor afectación del estado general. Dos días después, cursó somnoliento, febril en $38,5^{\circ} \mathrm{C}$, taquicárdico: 188 por min, cianótico y con disminución de saturación de oxígeno hasta $70 \%$. Se realizaron análisis mediante prueba rápida con antígeno NS1 que resultó positivo, además de IgG e IgM para dengue que resultaron negativos. Fue transferido al hospital de referencia en Huánuco, donde por diagnóstico probable de dengue y cultivos negativos se decidió suspender el tratamiento antimicrobiano, iniciándose medidas de soporte e hidratación con solución glucosada.

Durante su estadía hospitalaria, al cuarto día de enfermedad, la plaquetopenia se hizo progresivamente más intensa, llegando hasta 10.000 céls $/ \mathrm{mm}^{3}$, y con alteración del perfil de coagulación, por lo que se indicó transfusión de plasma fresco congelado. Además, presentó alteración de electrolitos y del perfil hepático (Tabla 1). Por distensión abdominal se realizó una ecografía, encontrándose líquido libre en la cavidad abdominal y la vesícula biliar edematosa. Se decidió continuar con manejo según las guías clínicas de tratamiento de dengue en pediatría de la OMS: hidratación, infusión de solución salina y uso de antipiréticos. Al sexto día del ingreso, el RN presentó un rash generalizado característico.

El paciente evolucionó con mejoría clínica y de la plaquetopenia, con disminución de las enzimas hepáticas, por lo que fue dado de alta dos días después. Posterior al alta, llegaron resultados confirmatorios de infección por dengue serotipo 2 (DENV-2), que se realizó mediante prueba de RPC en el Instituto Nacional de Salud de Perú.

\section{Discusión}

La enfermedad del dengue está muy bien descrita, tanto en la población adulta como pediátrica, con estudios multicéntricos que han sustentado guías y consensos de tratamiento 9 , los que se aplican en muchos países, incluyendo al Perú, a través de las normas técnicas del Ministerio de Salud. A pesar de ello, el dengue en la etapa neonatal aún sigue siendo una enfermedad poco diagnos-

\begin{tabular}{|c|c|c|c|c|c|c|c|}
\hline & D1* & D2 & D3 & D4 & D5 & D7 & D8 \\
\hline Hemoglobina (g/dl) & & 16,4 & 16 & 15,8 & 12,8 & & 10,6 \\
\hline Hematocrito (\%) & & 50,4 & 50,4 & 50,6 & 41 & & \\
\hline Leucocitos $\left(/ \mathrm{mm}^{3}\right)$ & 6.430 & 5.310 & 9.980 & & & & \\
\hline Baciliformes (\%) & 0 & 0 & 0 & & & & \\
\hline Segmentados (\%) & 69 & 40 & 55 & & & & \\
\hline Linfocitos (\%) & 17 & 47 & 31 & & & & \\
\hline Plaquetas $(x$ 1.000/mm³) & 220 & 60 & 27 & 25,5 & 10 & 98 & 98 \\
\hline Tiempo de protrombina & & & & & $18 \mathrm{seg}$ & 14 seg & $14 \mathrm{seg}$ \\
\hline Tiempo de tromboplastina & & & & & $123 \mathrm{seg}$ & $68 \mathrm{seg}$ & $58 \mathrm{seg}$ \\
\hline Proteína $\mathrm{C}$ reactiva & $+* *$ & 0,39 & & & & & \\
\hline $\mathrm{Na}(\mathrm{mEq})$ & & & 130 & & 143 & & \\
\hline $\mathrm{K}(\mathrm{mEq})$ & & & 6,7 & & 5,07 & & \\
\hline $\mathrm{LDH}(\mathrm{U} / \mathrm{I})$ & & & & & 2882 & & 2532 \\
\hline GOT (U/I) & & 87 & & & 652 & & 325 \\
\hline GPT (U/I) & & 28 & & & 244 & & 193 \\
\hline
\end{tabular}

ticada e informada y la mayor parte de las publicaciones al respecto son casos clínicos o pequeñas series de casos.

En el Perú, circulan actualmente los cuatro serotipos. La enfermedad del dengue data desde inicios de 1700 , cuando se describieron casos febriles compatibles con dengue clásico $^{3}$, pero no es hasta la epidemia de 1990 que se tipifica la presencia del virus serotipo 1 (DENV-1), siendo éste el primer serotipo identificado en el Perú; luego, en 1995 se identificó la presencia del DENV-2 genotipo americano, aunque la presentación de casos graves e incluso los casos fatales está asociado con el ingreso del DENV-2 genotipo americano/asiático, que sucedió a finales del año $2000^{10}$; en nuestro paciente no se realizó la identificación del genotipo específico de DENV-2.

En el Departamento de Huánuco, el año 2016 se detectaron 263 de un total nacional de 3.864 casos confirmados de dengue, presentando una tasa de incidencia de más de 30 casos por 100 mil habitantes en las provincias de Rupa-Rupa (lugar de donde provenía nuestro paciente), y Luyando, indicando que son zonas de hiperendemia. Además, estas mismas áreas presentan un índice aédico (porcentaje de casas con el mosquito Aedes aegypti) de casi 30\%, demostrando que es una zona de alto riesgo para la transmisión vectorial de la enfermedad ${ }^{4}$.

La tasa de transmisión vertical de dengue varía según los estudios. Arragain, en un seguimiento prospectivo de 10 mujeres, realizado en Nueva Caledonia ${ }^{11}$, mostró una tasa de transmisión vertical de $90 \%$; mientras que 
Ribeiro ${ }^{12}$ en Brasil, al evaluar 35 tejidos ovulares o placentarios de madres con dengue, describió siete casos de transmisión vertical (20\%). En una revisión retrospectiva de binomios madre-hijo en Guyana Francesa, Basurko ${ }^{13}$ encontró una tasa de transmisión de $15 \%$.

Se desconoce cuáles son los mecanismos patogénicos implicados en la transmisión vertical; no obstante, algunas hipótesis sugieren que el daño endotelial asociado a la permeabilidad vascular aumentada, los cuales son marcadores característicos de la enfermedad del dengue, podrían facilitar el paso de virus infectantes desde la circulación materna, a través de la barrera placentaria, al feto ${ }^{7}$.

En nuestro caso clínico no fue posible determinar el mecanismo especifico de transmisión. Sin embargo, a la luz de los elementos clínicos la transmisión vertical transplacentaria parece ser lo más probable, puesto que la madre empezó el cuadro clínico al cuarto día posterior al parto, lo que está dentro del tiempo de incubación establecido (4-8 días) ${ }^{1}$. Es probable que la madre estaba en condición de viremia antes del parto. El RN empezó su cuadro clínico a los 12 días de vida, límite de lo descrito (3-11 días) en que usualmente se inicia la enfermedad en neonatos ${ }^{8}$; sí está dentro del límite posterior a la enfermedad materna (0-8 días), cuando la transmisión vertical transplacentaria ha sido comprobada ${ }^{11-13}$.

Además, en nuestro paciente la posibilidad de transmisión vertical a través de la lactancia materna tiene que ser considerada. Esta vía de transmisión se planteó experimentalmente cuando se encontraron virus viables infectantes en la leche materna, con cultivo positivo realizado en líneas celulares ${ }^{14}$; sin embargo, en otro estudio posterior, pese a que se encontró la presencia del virus, los cultivos celulares de leche materna resultaron negativos ${ }^{11}$.

La mayoría de los casos clínicos se centran en demostrar la transmisión vertical, pero muy pocos evalúan la posibilidad de una infección primaria a esta edad. Aurpibul en Tailandia ${ }^{15}$, reportó el caso de una RN de 7 días de vida, que desarrolló un dengue grave con evolución favorable, sin encontrarse signos de enfermedad en la madre, pero con anticuerpos IgG e IgM positivos; mientras que en un estudio en China, Limei y cols. encontraron 12 pacientes

\begin{tabular}{|lcc|}
\hline \multicolumn{3}{l}{ Tabla 2. Evolución y pruebas diagnósticas en dengue neonatal } \\
\hline Inicio de síntomas* & Mínimo & Máximo \\
\hline Duración de la hospitalización & 30 min $^{16}$ & 29 días $^{16}$ \\
\hline Positividad de NS1** & 4 días $^{8.16}$ & 32 días $^{24}$ \\
Positividad de lgM** & $10 \mathrm{~h}^{17}$ & 8 días $^{25}$ \\
\hline Positividad de reacción de polimerasa en cadena (RPC)** & $8 \mathrm{~h}^{17}$ & 6 días $^{19}$ \\
\hline *Posterior al nacimiento. **Posterior al inicio de enfermedad & 4 días $^{22}$ \\
\hline
\end{tabular}

con dengue neonatal. De ellos, las madres tenían dengue confirmado o sospechado en nueve casos, mientras que en tres se descartó la presencia de dengue en la madre, por lo que se confirmó una infección primaria, con un inicio de síntomas hasta los 29 días de vida ${ }^{16}$.

Nuestro paciente, según la clasificación de la OMS, fue un dengue grave, por presentar daño grave de órganos, elevación de transaminasas y escape de líquidos, al encontrarse ascitis y vesícula edematosa y principalmente dificultad respiratoria9.

Los síntomas respiratorios están ampliamente descritos. Arragain describió hipoxemia en $40 \%$ de los $\operatorname{casos}^{11}$. En Brasil, un caso de los siete descritos comenzaron su enfermedad con dificultad respiratoria ${ }^{12}$, y en Guadalupe, dos de los cuatro casos descritos se iniciaron así17. De manera semejante, en el primer caso descrito en Taiwán, la dificultad respiratoria fue el motivo de ingreso a unidad de cuidados intensivos ${ }^{18}$.

En relación a los exámenes de laboratorio, todos los casos estudiados mostraron trombocitopenia, haciendo de este hallazgo un marcador de enfermedad; asimismo las alteraciones de coagulación, como en nuestro paciente, se repitieron en varios de los casos clínicos, así como el incremento de enzimas hepáticas que eran algunos de los

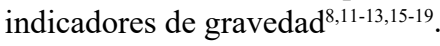

En nuestro caso clínico, el diagnóstico sospechado en el primer hospital fue una sepsis neonatal. Puesto que las definiciones coinciden en ambos casos, este hecho se repite en la mayor parte de los casos clínicos, en los cuales, como en nuestro paciente, se utilizaron antibacterianos ${ }^{8,12,16,17,20-26}$; incluso se han utilizado aun después de haberse confirmado el diagnóstico de dengue, por la sospecha de una infección bacteriana concomitante ${ }^{15}$. Otras arbovirosis, como el virus Chikungunya y Zika, este último ampliamente estudiado por las consecuencias congénitas de la transmisión transplacentaria, siempre tienen que ser sospechadas, puesto que su epidemiología, vector y manifestaciones clínicas son muy semejantes al dengue. Hasta el momento no se han descrito casos de estas arbovirosis en la zona de Huánuco y Tingo María 4 .

El diagnóstico de dengue neonatal varía en los distintos estudios, pero todos se basan en la determinación de pruebas de anticuerpos tipo $\operatorname{IgG}$ e $\operatorname{IgM}$, indicándose como confirmatorio si presenta ambos anticuerpos o una IgM positiva, para evitar el sesgo de la positividad de la IgG por atravesar la barrera placentaria, asociado a la presencia de antígeno NS1 ${ }^{8,15,16,17,19,20,24-27}$. Otros estudios además utilizaron prueba confirmatoria molecular mediante RPC ${ }^{11-13,18,21-23}$ e inmunohistoquímica ${ }^{12}$. Los tiempos de positividad, junto con algunos datos de evolución, se muestran en la Tabla 2.

El tratamiento que se realizó en nuestro paciente coincide con el utilizado en la mayor parte de los casos estudiados. En una importante proporción de pacientes se 
realizó transfusión de plasma fresco congelado y plaquetas, los que se indicaron en su mayoría, aun cuando no se conocía el diagnóstico de dengue ${ }^{13,15-18,21,23,25,28}$. En todas las revisiones se realizó manejo de soporte e hidratación, ya fuese con solución glucosada, y principalmente con solución fisiológica, con una evolución clínica favorable $8,11-13,15-29$.

La prevención del dengue se sustenta en dos pilares: inmunización, sin estudios ni indicaciones aun en población gestante; y el control vectorial, con eliminación adecuada de los residuos sólidos y la mejora de las prácticas de almacenamiento de agua, para evitar que los mosquitos hembras depositen sus huevos ${ }^{3}$. Este último es el factor más importante en disminuir la incidencia del dengue y con ello reducir el riesgo de transmisión tanto a mujeres gestantes y a los neonatos.

Además, debe diagnosticarse adecuadamente a toda mujer gestante con sospecha epidemiológica de dengue, con personal de salud capacitado en reconocer los síntomas y brindar una atención oportuna, realizando vigilancia en el neonato, durante las dos primeras semanas de vida ${ }^{21,26}$.
En conclusión, el dengue en la etapa neonatal debe ser siempre sospechado en áreas donde la enfermedad tiene carácter endémico y más aún en situaciones de epidemias. Además, deben proponerse modelos de actuación y guías de práctica clínica en este grupo de pacientes.

\section{Resumen}

El dengue es la enfermedad transmitida por artrópodos de mayor importancia en salud pública; sin embargo, su presencia en la etapa neonatal está pobremente estudiada, con pocos casos descritos en el mundo. Se presenta el caso clínico de un recién nacido de 12 días de vida, con historia de fiebre e irritabilidad, por lo que se sospechó una sepsis neonatal. Por trombocitopenia progresiva y el antecedente de la madre con diagnóstico de dengue posterior al parto, se diagnosticó un dengue en el RN por test rápido y confirmado por biología molecular, con una evolución favorable. El dengue en la etapa neonatal debe ser sospechado en áreas endémicas y proponerse guías de manejo.

\section{Referencias bibliogáficas}

1.- Guzmán M G, Harris E. Dengue. Lancet 2015; 385: 453-65.

2.- $\quad$ Bhatt S, Gething P W, Brady O J, Messina J P, Farlow A W, Moyes C L, et al. The global distribution and burden of dengue. Nature 2013; 496: 504-7.

3.- Cabezas C, Fiestas V, García-Mendoza M, Palomino M, Mamani E, Donaires F. Dengue en el Perú: a un cuarto de siglo de su reemergencia. Rev Peru Med Exp Salud Publica 2015; 32: 146-56.

4.- Dirección General de Epidemiología (DGE). Sala Situacional. [Internet] Ministerio de Salud. Lima, Perú. 2017. http://www.dge.gob.pe/ portal/docs/vigilancia/sala/2017/salaSE07.pdf. [Fecha de acceso: 28 de febrero de 2017].

5.- Halstead S B, Lan N T, Myint T T, Shwe T N, Nisalak A, Kalyanarooj S, et al. Dengue hemorrhagic fever in infants: research opportunities ignored. Emerg Infect Dis 2002; 8: 1474-9.

6.- Jain A, Chaturvedi U C. Dengue in infants: an overview. FEMS Immunol Med Microbiol 2010; 59: 119-30.

7.- Paixão E S, Teixeira M G, Costa M D C, Rodrigues L C. Dengue during pregnancy and adverse fetal outcomes: a systematic review and meta-analysis. Lancet Infect Dis 2016; 16 : 857-65.

8.- $\quad$ Salgado D M, Rodríguez J A, Lozano L del P, Zabaleta T E. Dengue perinatal. Biomédica 2013; 33 Supl 1: 14-21.
9.- Organización mundial de la salud. Dengue: Guías para el diagnóstico, tratamiento, prevención y control. OMS 2009. http://www. bvs.hn/Honduras/salud/dengue.guias.para. el.diagnostico.tratamiento.prevencion.y.control. pdf

10.- Mamani E, Álvarez C, García M M, Figueroa D, Gatti M, Guio H, et al. Circulación de un linaje diferente del virus dengue 2 genotipo América/Asia en la región amazónica de Perú, 2010. Rev Peru Med Exp Salud Publica 2011; 28: 72-7.

11.- Arragain L, Dupont-Rouzeyrol M, O'Connor O, Sigur N, Grangeon J P, Huguon E, et al. Vertical transmission of dengue virus in the peripartum period and viral kinetics in newborns and breast milk: new data. J Pediatric Infect Dis Soc 2016. pii: piw058. Disponible en https://doi.org/10.1093/jpids/piw058. [Fecha de acceso: 28 de febrero de 2017].

12.- Ribeiro C F, Lopes V G, Brasil P, Coelho J, Muniz A G, Noqueira R M. Perinatal transmission of dengue: a report of 7 cases. $\mathrm{J}$ Pediatr 2013; 163: 1514-6.

13.- Basurko C, Carles G, Youssef M, Guindi W E. Maternal and fetal consequences of dengue fever during pregnancy. Eur J Obstet Gynecol Reprod Biol 2009; 147: 29-32.

14.- Barthel A, Gourinat A C, Cazorla C, Joubert C, Dupont-Rouzeyrol M, Descloux E. Breast milk as a possible route of vertical transmission of dengue virus? Clin Infect Dis 2013; 57: 415-7.

15.- Aurpibul L, Khumlue $P$, Issaranggoon na ayuthaya S, Obedorfer P. Dengue shock syndrome in an infant. BMJ Case Rep 2014. Jul 29. pii:bcr2014205621. Disponible en https://www.ncbi.nlm.nih.gov/pmc/articles/ PMC4120039/pdf/bcr-2014-205621.pdf. [Fecha de acceso: 28 de febrero de 2017].

16.- Tan L, Wang J, Zeng F, Zhang Y, Fang C, Nie $\mathrm{C}$, et al. Analysis of clinical characteristics of the 12 cases of neonatal dengue fever in Guangzhou in 2014 and literatures review. Zhonghua Er Ke Za Zhi 2015; 53: 943-7.

17.- Mazarin N, Rosenthal J M, Devenge J. Motherinfant dengue transmission during the 20092010 dengue epidemic: report of four cases. Arch Pédiatr 2014; 21: 745-9.

18.- Yang S T, Chen H L, Yeh C T, Lee W T. Vertical transmission of dengue fever: First case reported in Taiwan. J Formos Med Assoc 2015; 114: 558-9.

19.- Ekanayake C D, Padumadasa S, Premaratna R, Rajendrajith S, Samaranayake W W. Transplacental transfer of dengue. Ceylon Med J 2014; 59: 145-6.

20.- Sinhabahu V P, Sathananthan R, Malavige G N Perinatal transmission of dengue: a case report. BMC Res Notes 2014; 7: 795.

21.- Berberian G, Fariña D, Rosanova M T, Hidalgo S, Enría D, Mitchenko A, et al. Perinatal dengue infection. Arch Argent Pediatr 2011; 109: 232-6.

22.- Silva Delgado H, Ruiz Ríos J C, Vela Barbarán E L, Rengifo Del Aguila D, García M M, Rodríguez Benavides L, et al. Dengue neonatal en el Perú: reporte de un caso. Rev Peru Med Exp Salud Publica 2011; 28: 140-4. 
23.- Maroun S L, Marliere R C, Barcellus R C, Barbosa C N, Ramos J R, Moreira M E. Case report: vertical dengue infection. J Pediatr (Rio J) 2008; 84: 556-9.

24.- Romero-Santacruz E, Lira-Canul J J, PachecoTugores F, Palma-Chan A G. Dengue neonatal. Presentación de casos clínicos. Ginecol Obstet Mex 2015; 83: 308-15.

25.- Fajardo-Ochoa F, De la Re-Montaño N, Araiza-Martínez CU. Dengue neonatal, reporte de un caso. Bol Clin Hosp Infant Edo Son 2015; 32: 40-3.

26.- Balcázar Rincón L E, Ramírez Alcántara Y L. Dengue neonatal: a propósito de un caso. Rev Pediatr Aten Primaria. 2016; 18 : 217-21.

27.- Brítez S, Mir R, Lacarrubba J, Mendieta E, Céspedes E, Genes L. Dengue de transmisión vertical: revisión de una serie de casos. Pediatr (Asunción) 2014; 41: 25-31.
28.- Fonseca-Becerra C E, Bayona-Ospina M A. Dengue en recién nacidos: presentación de dos casos en Neiva (Huila, Colombia) y revisión de la literatura. Rev Colomb Obstet Ginecol 2010; 61: 72-7.

29.- Camacho Ramírez R I, Ávila Reyes R, Sánchez Zapata M H, Guerrero Méndez N A, Brusollo Ceballos R, Herrera P M, et al. Dengue neonatal. Reporte de un caso. Enf Infec Microbiol 2014; 34: 155-9. 